\title{
MicroRNA-214 acts as a potential oncogene in breast cancer by targeting the PTEN-PI3K/Akt signaling pathway
}

\author{
FANG WANG, LIN LI, ZHUO CHEN, MINGZHI ZHU and YUANTING GU \\ The Second Department of Breast Surgery, The First Affiliated Hospital \\ of Zhengzhou University, Zhengzhou, Henan 450052, P.R. China
}

Received February 9, 2015; Accepted February 25, 2016

DOI: $10.3892 /$ ijmm.2016.2518

\begin{abstract}
Breast cancer ranks as the leading cause of cancerrelated mortality in females worldwide. It has been proven that microRNAs (miRNAs or miRs), a type of non-coding RNA, are involved in tumorigenesis. An increasing number of studies has confirmed the critical role of miR-214 in certain types of cancer. Nevertheless, the biological function of miR-214, as well as its underlying mechanisms of action in breast cancer remain largely unknown. In the present study, the expression of miR-214 was found to be upregulated in four human breast cancer cell lines in contrast to its expression level in the non-malignant breast epithelial cell line, MCF-10A. Moreover, the overexpression of miR-214 markedly increased cell viability and abrogated the apoptosis triggered by serum starvation, indicating that miR-214 plays a pivotal role in breast cancer cell growth. Further analysis suggested that the upregulation of miR-214 markedly induced the activation of the phosphoinositide 3-kinase (PI3K)/Akt signaling pathway, which largely accounted for the protective effects of miR-124 on cancer cell growth. This was further confimed by pre-treatment with the PI3K/Akt inhibitor, LY294002, which markedly attenuated the miR-214-induced increase in cell viability and resistance to apoptosis. Furthermore, the expression of phosphatase and tensin homolog (PTEN) was decreased following transfection wtih miR-214 mimics and PTEN was confirmed as the direct target of miR-214 by bioinformatics analysis and a dual-firefly luciferase reporter assay. Importantly, the introduction of PTEN cDNA lacking the 3' untranslated region (3'UTR) significantly inhibited the miR-214-induced activation of the PI3K/Akt signaling pathway, and abrogated the protetive effects of miR-214 on cell survival and resistance to apoptosis. Taken together, these findings suggest that miR-214 possesses oncogenic activity and that its effects are medi-
\end{abstract}

Correspondence to: Dr Yuanting Gu, The Second Department of Breast Surgery, The First Affiliated Hospital of Zhengzhou University, 1 Jianshe East Road, Zhengzhou, Henan 450052, P.R. China E-mail: yuantinggu@163.com

Key words: breast carcinoma, microRNA-214, cell viability, resistance to apoptosis, phosphatase and tensin homolog, phosphoinositide 3-kinase/Akt pathway ated through the promotion of cell growth by targeting the PTEN-PI3K/Akt pathway. Thus, pharmaceutical interventions targeting miR-124 may provide a promising therapeutic strategy for the treatment of breast cancer.

\section{Introduction}

Breast cancer is the most common type of cancer affecting females worldwide, and ranks as the second most common type of cancer with 1.38 million new cases diagnosed and 458,100 associated deaths in 2008 (1). Despite advances in the diagnosis and the implementation of novel strategies for the treatment of breast cancer, interventions are often ineffective due to the high proliferative ability of cancer cells and intrinsic resistance to clinical therapies, including chemotherapy (2). Therefore, there is an urgent need to develop novel therapeutic strategies by elucidating the molecular mechanisms responsible for the development and progression of breast cancer.

MicroRNAs (miRNAs or miRs) are a class of small (21-24 nucleotides), non-coding RNA molecules which function as pivotal regulators of gene expression by interacting with the 3'-untranslated region (3'UTR) of their target mRNA. miRNAs have been demonstrated to regulate thousands of human genes and are of fundamental importance in various human diseases, such as cardiovascular disease, Alzheimer's disease and autoimmune diseases $(3,4)$. Several studies have confirmed the abnormal expression of miRNAs in several types of cancer, including colon cancer, hepatocellular carcinoma, lung cancer and breast cancer (5-7). Emerging evidence has indicated that miRNAs are capable of modulating various biological processes which occur in cancer cells, such as cell proliferation, invasion, cell cycle arrest and survival $(8,9)$. Of note, many miRNAs are located at fragile sites or cancer-associated regions, which may explain the reason for the correlation between tumorigenesis and the aberrant expression of certain miRNAs. Among these, miR-214 has been demonstrated to be dysregulated in several types of human malignancies $(8,10,11)$. However, miR-214 plays various roles, which may even be opposing roles, in different types of cancer. In hepatoma, miR-214 is downregulated and acts as an anti-oncogene, affecting hepatoma cancer cell growth, metastasis and tumor angiogenesis $(10,12)$. By contrast, miR-214 is significantly upregulated in osteosarcoma tissues with a large tumor size and positive metastasis, and it is recognized as an independent prognostic factor of unfavorable survival 
in pediatric osteosarcoma (11). The serum concentrations of miR-214 have been proven to be significantly higher in patients with breast cancer in contrast to those in healthy women (13). However, the role of miR-124 in breast cancer is unclear as its underlying mechanisms of action remain poorly understood.

In the present study, the expression level of miR-214 in breast cancer cell lines was determined. Importantly, we examine the effects of miR-214 on cell proliferation and resistance to apoptosis. Furthermore, the underlying mechanisms of action of miR-214, as well as its potential targets were also investigated.

\section{Materials and methods}

Reagents and antibodies. The phosphoinositide 3-kinase (PI3K) inhibitor, LY294002 (\#440202), was purchased from Calbiochem (San Diego, CA, USA). The polyclonal antibodies against human Akt (\#9272) and phosphorylated (p-)Akt (\#9271) were obtained from Cell Signaling Technology (Beverly, MA, USA). The antibodies against Bcl-2 (\#sc-509) were purchased from Santa Cruz Biotechnology, Inc. (Santa Cruz, CA, USA). Rabbit polyclonal antibodies against cyclin D1 (ab15196) and p27 (ab7961) was purchased from Abcam (Cambridge, UK). The antibodies against phosphatase and tensin homolog (PTEN; \#9559) were purchased from Cell Signaling Technology (Danvers, MA, USA).

Human cancer cell lines and cell culture. The human breast cancer cell lines, MCF-7, MDA-MB-231, MDA-MB-453 and T47D, were obtained from the American Type Culture Collection (ATCC; Manassas, VA, USA). The non-malignant breast epithelial cell line, MCF-10A, was also from ATCC and was used as the 'normal' control for human breast cancer cell analysis. The above-mentioned 4 breast cancer cell lines were grown in RPMI-1640 medium supplemented with $10 \%$ fetal calf serum. The MCF-10A cells were cultured in Mammary Epithelial Cell Basal Medium (Cambrex, Walkersville, MD, USA). All media were supplemented with $2 \mathrm{mM}$ glutamine, $100 \mu \mathrm{g} / \mathrm{ml}$ streptomycin and penicillin. The cells were all incubated in a humidified atmosphere at $37^{\circ} \mathrm{C}$ with $5 \% \mathrm{CO}_{2}$.

RNA extraction and reverse transcription-quantitative polymerase chain reaction $(R T-q P C R)$. The expression of miR-214 was analyzed using the TaqMan ${ }^{\circledR}$ microRNA reverse transcription kit (Applied Biosystems, Foster City, CA, USA). Briefly, total RNA was extracted from the cultured cells using TRIzol reagent (Invitrogen, Carlsbad, CA, USA) according to the manufacturer's instructions. The resulting total RNA was then used for the primer-specific reverse transcription of miR-214 and U6. To analyze the expression of miR-214 in breast cancer cells, approximately $2 \mu \mathrm{l}$ cDNA was subjected to qPCR using a 7900HT Fast Real-Time PCR System (Applied Biosystems). The reaction conditions and procedures were performed according to the instructions provided with the TaqMan microRNA reverse transcription kit (Applied Biosystems). U6 was used to normalize the miRNA. All results were calculated using the $2^{-\Delta \Delta C t}$ method.

Oligonucleotide transfection. The miR-214 mimics and scramble control miRNA (miR-con) sequences were used as previously described (14). All the oligonucleotides were obtained from and purified by RiboBio (Guangzhou, Guangdong, China). For transfection, approximately $1 \times 10^{5}$ cells were seeded into 12-well plates. When the cells reached $50-80 \%$ confluence, 0.4 nmol miRNA mimics or miR-con were mixed with $15 \mu \mathrm{l}$ GenePORTER 2 Transfection Reagent (GTS, San Diego, CA, USA). The above mixture was then separately transfected into the cells. Following incubation for $6 \mathrm{~h}$, fresh medium was added for a further $48 \mathrm{~h}$. The transfection efficiency of the miR-214 mimics was evaluated using RT-qPCR.

Construction and transfection of PTEN expression vectors. To obtain the PTEN expression vectors, wild-type PTEN lacking the 3'UTR region was cloned into the pcDNA3.1 vector by Genesil Biotechnology Co. Ltd. (Wuhan, China). When the cells grew to $70-80 \%$ confluency, $15 \mu \mathrm{g}$ of pcDNA-PTEN or pcDNA3.1 empty vector were transfected into the MCF-7 cells using Lipofectamine 2000 (Invitrogen) in accordance with the manufacturer's instructions. Following incubation for $6 \mathrm{~h}$, the medium was replaced with the fresh RPMI-1640 medium containing $10 \%$ fetal bovine serum (FBS). The transfected cells were evaluated by western blot analysis.

Dual-luciferase reporter assay in vitro. For luciferase reporter experiments, the 3'UTR sequence of PTEN predicted to interact with miR-214 or a mutated sequence within the predicted target sites was synthesized and inserted into the MluI and HindIII sites of a pGL3 vector (Promega, Madison, WI, USA). These constructs were known as pGL3-PTEN-3'UTR-wt or pGL3-PTEN-3'UTR-mut, respectively. For the reporter assay, the MCF-7 cells were plated onto 12-well plates, and then co-transfected with the above-mentioned constructs and $5 \mathrm{ng}$ of pRL-TK (Promega), with or without miR-214 or miR-con using Lipofectamine 2000 reagent (Invitrogen). Approximately $48 \mathrm{~h}$ later, the cells were harvested. The luciferase and Renilla signals were determined using the Dual-Luciferase Reporter Assay system (Promega) according to the manufacturer's instructions.

Cell viability assay. The cells were seeded in a 96-well culture plate $\left(1 \times 10^{5}\right.$ cells/well) and allowed to attach for $24 \mathrm{~h}$. Following pre-treatment with the PI3K/Akt inhibitor, LY294002, the cells were transfected as described above. The culture medium was removed from each well and replaced with fresh medium containing $5 \mathrm{mg} / \mathrm{ml}$ MTT solution (Sigma-Aldrich, St. Louis, $\mathrm{MO}$, USA). The plate was then incubated at $37^{\circ} \mathrm{C}$ for an additional $5 \mathrm{~h}$. After removing the remaining supernatant, $100 \mu \mathrm{l}$ of DMSO were added and mixed thoroughly to dissolve the formed formazan crystals. Cell viability was then analyzed by measuring the absorbance of each well at $570 \mathrm{~nm}$. Relative cell viability was calculated as the absorbance percentage of the treatment group relative to the control group.

Analysis of cell apoptosis using flow cytometry (FCM). Cell apoptosis was detected by FCM. Briefly, following incubation with RPMI-1640 medium without serum for $24 \mathrm{~h}$, the transfected cells were pre-treated with $20 \mu \mathrm{M}$ LY294002. The cells were trypsinized and washed with PBS. The cells were then centrifuged at $1,000 \mathrm{x} \mathrm{g}$ for $10 \mathrm{~min}$ at room temperature and resuspended with $500 \mu \mathrm{l}$ of binding buffer, followed by incu- 
bation with $5 \mu \mathrm{l}$ Annexin V-FITC and $5 \mu \mathrm{l}$ PI (Sigma-Aldrich, Carlsbad, CA, USA) for $15 \mathrm{~min}$ at room temperature. All specimens were analyzed on a FACScan flow cytometer (BD Biosciences, Franklin Lakes, NJ, USA) for the relative quantification of apoptosis.

Western blot analysis. Total protein from the treatment groups was extracted using RIPA lysis buffer (Beyotime, Nantong, China) and quantified using a BCA assay kit (Pierce, Rockford, IL, USA). A total of $200 \mu \mathrm{g}$ of protein was separated by SDS-PAGE and transferred onto PVDF membranes (Schleicher \& Schuell GmbH, Dassel, Germany). After blocking with $5 \%$ non-fat milk, the membranes were incubated with antibodies against p-Akt, Akt, cyclin D1, p27, Bcl-2 and PTEN. Incubation with the primary antibody was carried out overnight at $4^{\circ} \mathrm{C}$. The membranes were then incubated with a secondary antibody conjugated to horseradish peroxidase (HRP; Jackson ImmunoResearch, West Grove, MA, USA). The LumiGLo reagent (KPL Inc., Gaithersburg, MD, USA) was introduced to visualize the bound antibodies. The protein expression levels were normalized by $\beta$-actin. The intensity of protein expression was quantified with ImageJ software (National Institutes of Health, Bethesda, MD, USA).

Statistical analysis. All results are presented as the means \pm SEM. The statistical significance of the differences between groups was analyzed using a Student's t-test. A P-value $<0.05$ was considered to indicate a statistically significant difference.

\section{Results}

Expression of miR-214 is increased in breast cancer cell lines. It has been demonstrated that miR-214 plays an important role in the progression of different types of cancer $(8,14)$. However, research into the role of miR-214 in breast cancer remains limited. To clarify this issue, in this study, miR-214 expression in 4 human breast cancer cell lines (MCF-7, MDA-MB-231, MDA-MB-453 and T47D) was assessed. Compared with the non-malignant breast epithelial cell line, MCF-10A, the marked upregulation of miR-214 expression was found in the 4 breast cancer cell lines (Fig. 1). Consequently, these results confirmed the notable upregulation of miR-214 in breast cancer cells, which may be important in the development and progression of human breast cancer. Furthermore, the relative lower expression of miR-214 was observed in the breast cancer MCF-7 cells in contrast to the other 3 breast cancer cell lines. Thus, to better investigate the effects of miR-214 upregulation in breast cancer cells, the MCF-7 cells were selected for use in the subsequent experiments.

miR-214 overexpression enhances cell viability. To explore the role of miR-214 in the development and progression of breast cancer, we evaluated the effects of the overexpression of miR-214 on MCF-7 cell growth. To determine the direct contribution of miR-214 in breast cell growth, we successfully induced the expression of miR-214 in MCF-7 cells by transfecting the cells with miR-214 mimics which were detected using RT-qPCR (Fig. 2A). Importantly, MTT assay confirmed that the overexpression of miR-214 markedly induced a

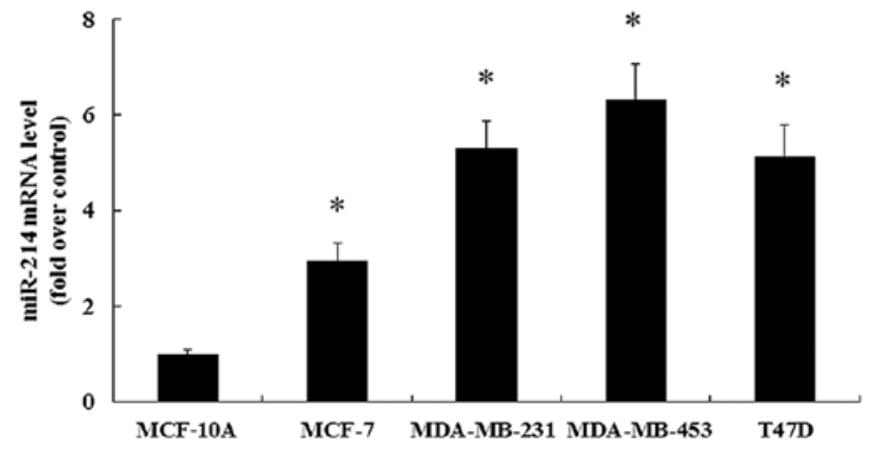

Figure 1. Expression of miR-214 in human breast cancer cell lines. To detect the expression levels of miR-214 in breast cancer cells, 4 human breast cancer cell lines (MCF-7, MDA-MB-231, MDA-MB-453 and T47D) were collected. The non-malignant breast epithelial cell line, MCF-10A, was used as a control. The expression of miR-214 was analyzed by RT-qPCR. ${ }^{*} \mathrm{P}<0.05$ vs. MCF-10A cells.
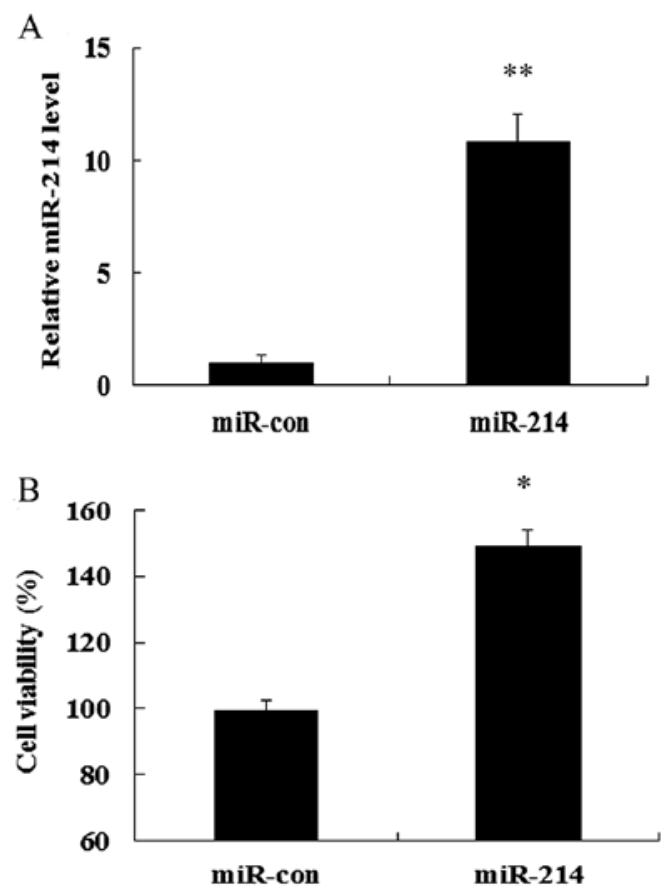

Figure 2. Upregulation of miR-214 promotes breast cancer cell survival. Following transfection with miR-214 mimics and scramble control miRNA (miR-con), (A) the corresponding transfection efficiency was evaluated by RT-qPCR). (B) The effect of miR-214 overexpression on cell viability was determined by MTT assay. ${ }^{*} \mathrm{P}<0.05$ and ${ }^{* *} \mathrm{P}<0.01$ vs. miR-con.

1.49-fold increase in cell viability, compared with that in the control group (transfected with miR-con; Fig. 2B). Therefore, these results indicate that the overexpressoin of miR-214 markedly enhances cell viability.

Overexpression of miR-214 abrogated cell apoptosis induced by serum starvation. To further examine the effect of miR-214 on cell apoptosis induced by serum starvation, Annexin V-FITC and PI staining was used. As shown in Fig. 3A, a notable increase in the cell apoptotic rate was observed when the cells were exposed to serum starvation conditions. However, transfection with miR-214 mimics markedly abrogated the increase in cell apoptosis, compared with that in the control groups (untreated control and miR-con-transfected cells). Further quantitative 
A

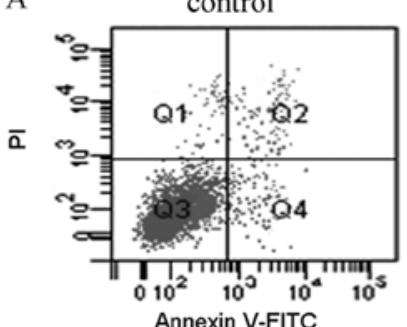

starved + miR-con

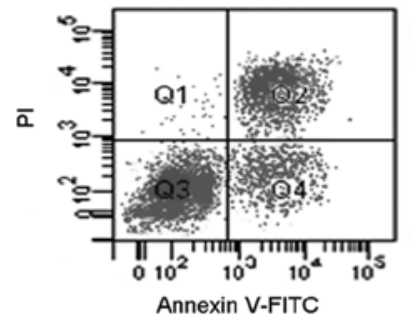

starved

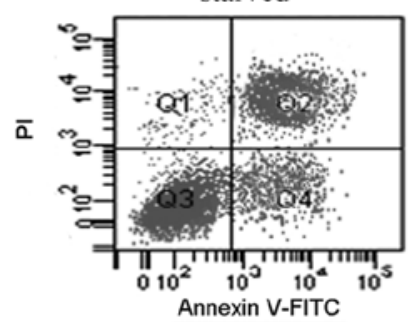

starved+miR-214

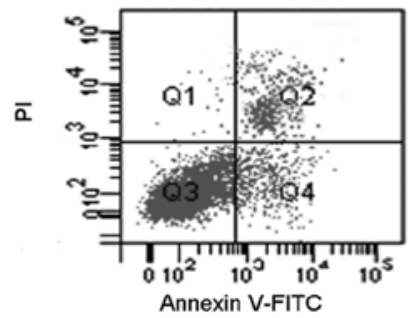

B

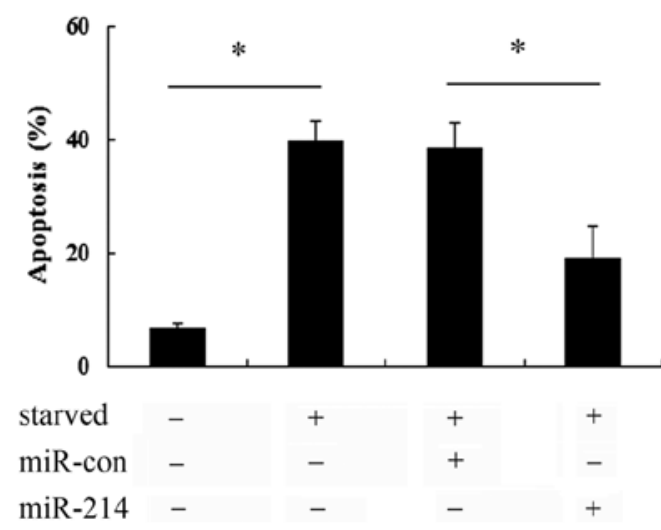

Figure 3. Effect of the overexpression of miR-214 on cell apoptosis. Following transfection with miR-214 mimics or scramble control miRNA (miR-con), the cells were cultured in RPMI-1640 medium without serum for $24 \mathrm{~h}$. The cells were then stained with $5 \mu 1$ Annexin V-FITC and $5 \mu 1$ PI. (A) The effect of miR-214 on cell apoptosis was further determined by flow cytometric analysis. (B) Corresponding quantitative analysis. "P $<0.05$. Q1, necrotic cells; Q2, late apoptotic cells; Q3, surviving cells; Q4, early apoptotic cells.

analysis revealed that serum starvation induced a 0.39 -fold increase in the cell apoptotic rate, which was markedly abrogated when the cells were transfected with miR-214 mimics (Fig. 3B), implying that miR-214 exerts protective effects against the apoptosis of breast cancer cells, thus playing a critical role in the development of breast cancer cells.

miR-214 exerts a positive effect on cell growth by regulating the activation of the PI3K/Akt pathway. It has been demonstrated that PI3K/Akt signaling plays a critical role in the development of cancer and multiple physiological processes (15). Thus, in order to elucidate the mechanisms responsible for the promoting effects of miR-214 on cell growth, the activation of the PI3K/Akt pathway was examined. As shown in Fig. 4A, transfection with miR-214 mimics markedly induced the expression of p-Akt, but not that of Akt. Moreover, the expression of the cell cycle inhibitory protein, p27, was notably decreased when the cells were transfected with miR-214 mimics, whereas the expression of cyclin D1 was increased. Furthermore, the overexpression of miR-214 induced a 2.46-fold increase in the expression of

the anti-apoptotic protein, Bcl-2 (Fig. 4B). Thus, these findings prompted us to hypothesize that the PI3K/Akt signaling pathway is associated with the promoting effects of miR-214 on the growth of breast cancer cells. Following pre-treatment with the PI3K inhibitor, LY294002, the miR-214-induced activation of the PI3K/Akt signaling pathway was markedly abrogated (Fig. 5A and B). Of note, pre-treatment with LY294002 markedly attenuated the miR-214-induced increase in cell viability (Fig. 5C). Importantly, miR-214 exerted a protective effect agasint cell apoptosis induced by serum starvation; this effect was also abrogated when the cells were pre-treated with LY294002 (Fig. 5D). Taken together, these data suggest that miR-214 exerts a protective effect against the apoptosis of breast cancer cells and promotes their growth, mainly by regulating the activation of the PI3K/Akt signaling pathway.

PTEN is a target of miR-214. It has been confirmed that PTEN negatively regulates PI3K/Akt signaling $(16,17)$. Thus, to further elucidate the mechanisms involved in the miR-214-mediated activation of the PI3K/Akt signaling pathway, we analyzed the expression of PTEN. As shown in Fig. 6A, a high PTEN expression was detected in the MCF-7 cells. However, transfection with miR-214 induced a marked downregulation (0.48-fold) in PTEN expression (Fig. 6B). It has been documented that miRNAs negatively regulate the expression of their targets, primarily by interacting with the 3'UTR of their mRNA, which ultimately leads to mRNA degradation or translational inhibition $(8,9)$. Further bioinformatics analysis indicated that there was a conservative binding site for miR-214 in PTEN (Fig. 6C). The 3'UTR sequence of PTEN which was predicted to interact with miR-214 was further synthesized and inserted into a pGL3 vector and separately termed pGL3-PTEN-3'UTR-wt and pGL3-PTEN-3'UTR-mut. Co-transfection of the MCF-7 cells with miR-214 and pGL3-PTEN-3'UTR-wt significantly suppressed the luciferase activity; however, this effect was not observed in the pGL3-PTEN-3'UTR-mut-trasnfected group (Fig. 6D). Taken together, these results confirmed that miR-214 downregulated PTEN expression by directly binding to the 3'UTR of PTEN mRNA in breast cancer cells.

PTEN is responsible for the promoting effects of $\mathrm{miR}-214$ on the survival and resistance to apoptosis of breast cancer cdells through PI3K/Akt signaling. To determine whether PTEN is repsonsible for the promoting effects of miR-214 on cell survival and the protective effects of this miRNA on the apoptosis of breast cancer cells, as well as the involvement of the PI3K/Akt signaling pathway, the expression of PTEN was ectopically induced by transfecting the cells with cDNA that contained only the coding region of PTEN, which should escape regulation by $\mathrm{miR}-214$ and thus, inhibit the function of miR-214. Following transfection of pcDNA-PTEN lacking 3'UTR into the MCF-7 cells, the expression of PTEN which had been inhibited by miR-214, was markedly increased; however, the increase in the levels of p-Akt induced by miR-214 was clearly abrogated (Fig. 6E), implying that miR-214 regulates the activation of the PI3K/Akt signaling pathway by targeting PTEN. Importantly, the overexpression of PTEN significantly abrogated the protective effects of miR-214 on cell survival (Fig. 6F). Simultaneously, PTEN expression also 
A

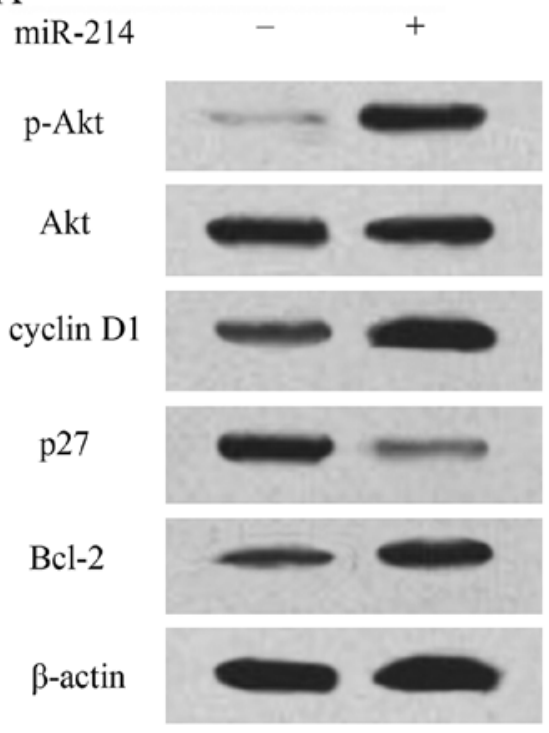

B

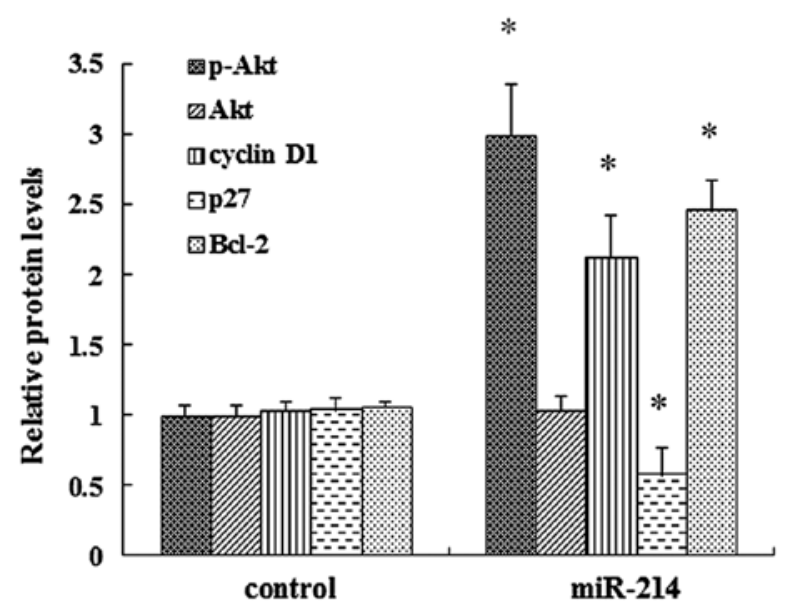

Figure 4. miR-214 induces the activation of the PI3K/Akt signaling pathway. MCF-7 cells were first infected with miR-214 mimics, or scramble control miRNA. (A) The activation of phosphorylated (p-)Akt and the corresponding downstream targets levels of cyclin D1, p27 and Bcl-2 were then examined by western blot analysis. (B) Corresponding quantitative analysis using Image J software. ${ }^{*} \mathrm{P}<0.05$ vs. control.

A

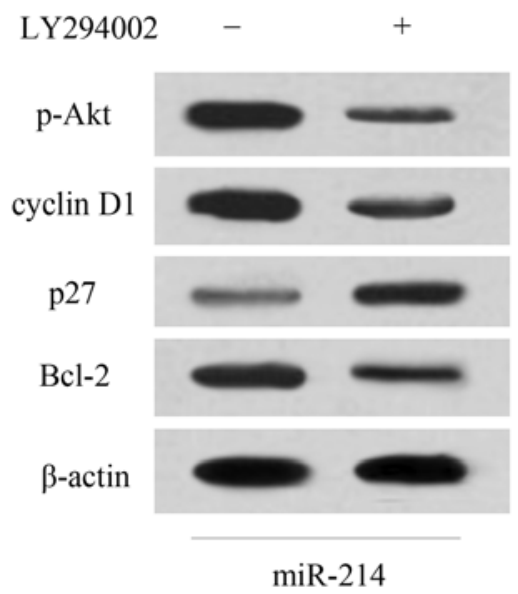

$\mathrm{C}$

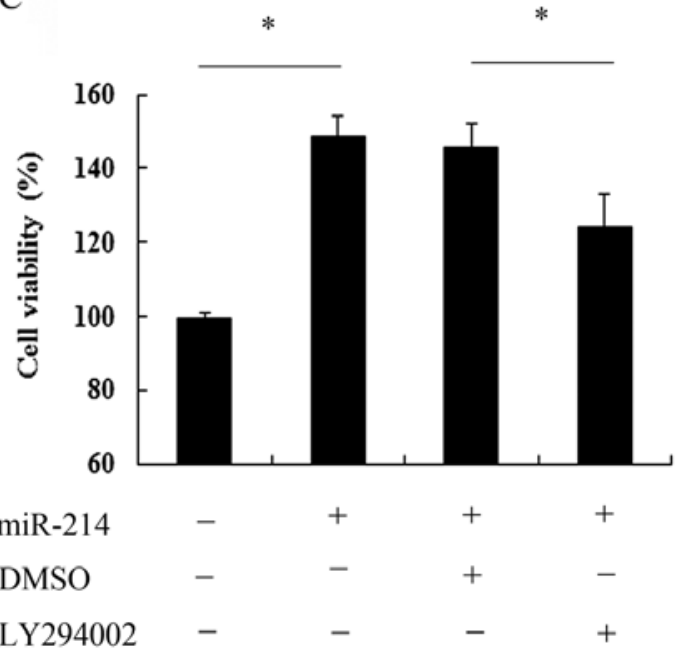

B

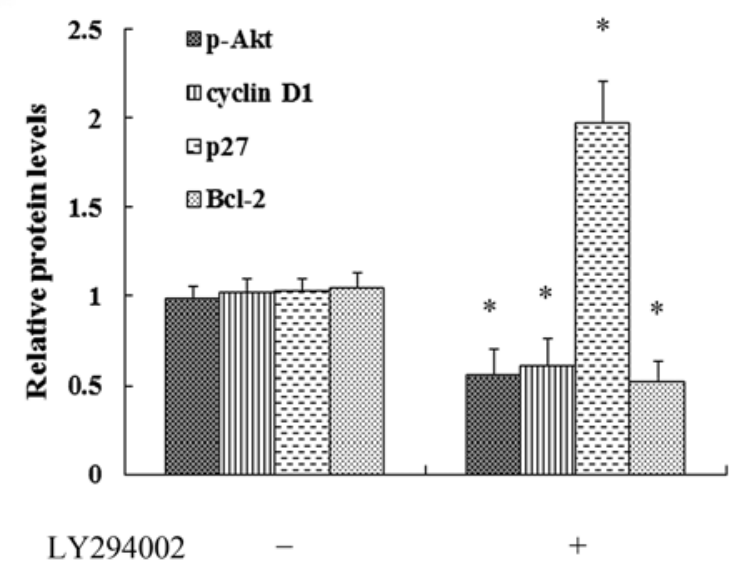

D
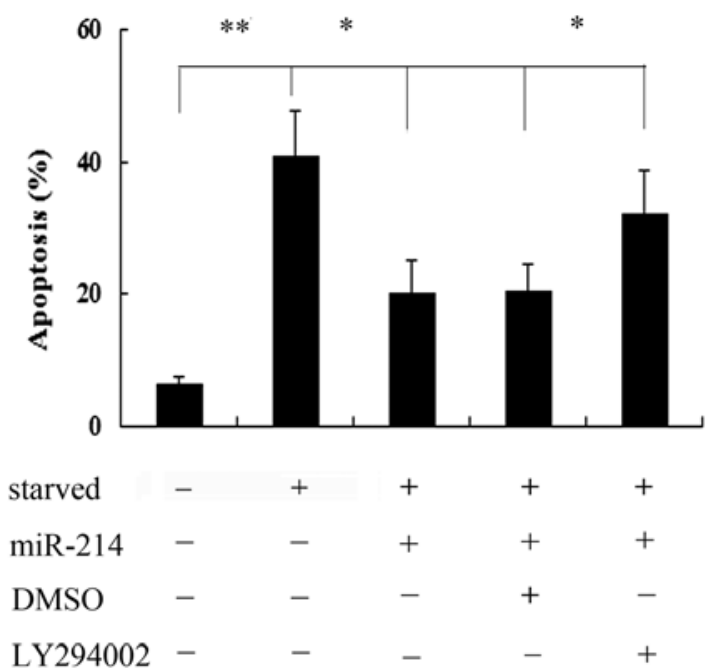

Figure 5. miR-214 increases cell viability and promotes resistance to apoptosis through PI3K/Akt signaling. The cells were pre-treated with the PI3K inhibitor, LY294002, and then transfected with miR-214 mimics. (A and B) The activation of the PI3K/Akt pathway was further examined by western blot analysis. The effects of LY294002 on miR-214-mediated (C) cell viability and (D) cell apoptosis were subsequently examined by MTT assay and Annexin V-PI staining, respectively. ${ }^{*} \mathrm{P}<0.05,{ }^{* *} \mathrm{P}<0.01$. 
A

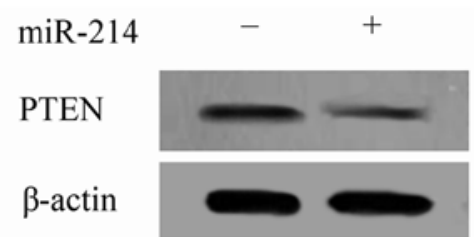

$\mathrm{C}$

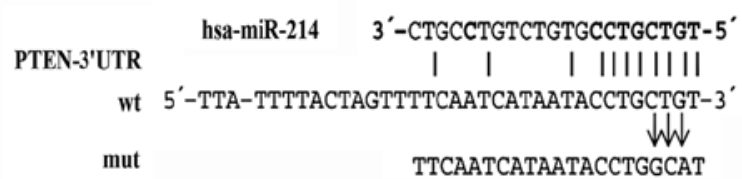

D

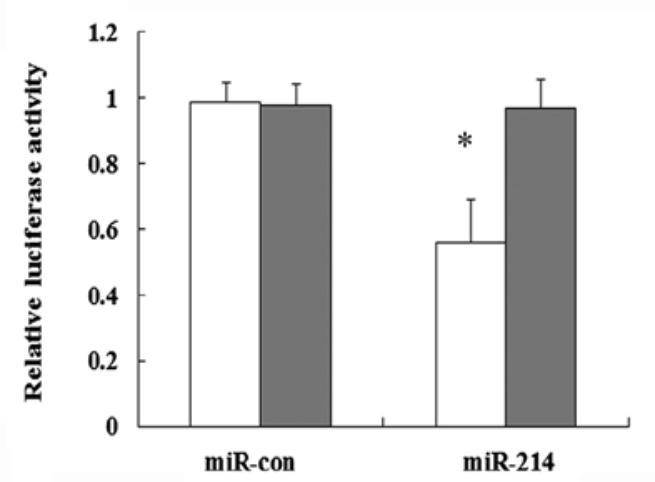

口pGL3-PTEN-3'UTR-wt घpGL3-PTEN-3'UTR-mut

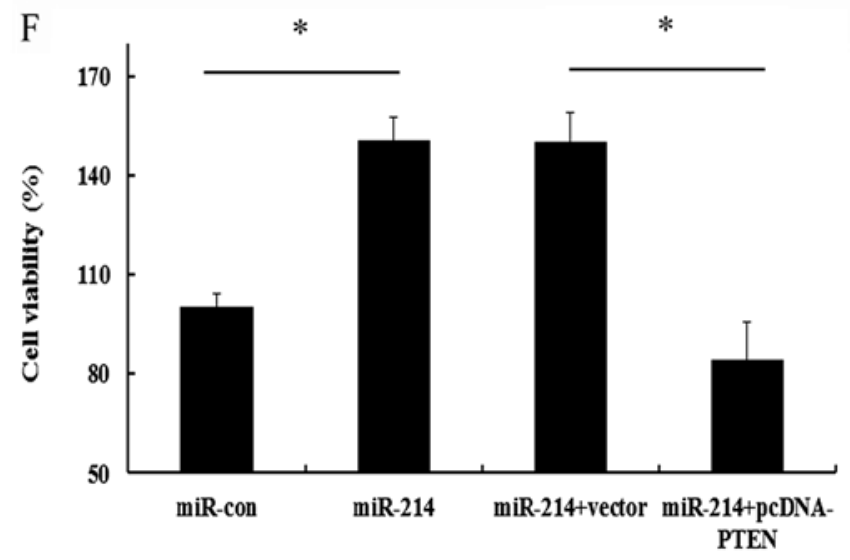

B

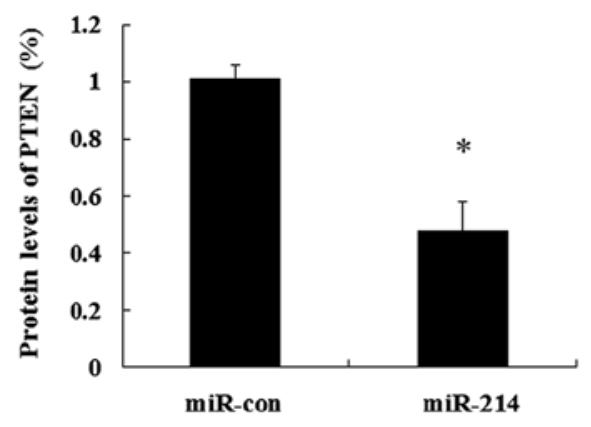

E

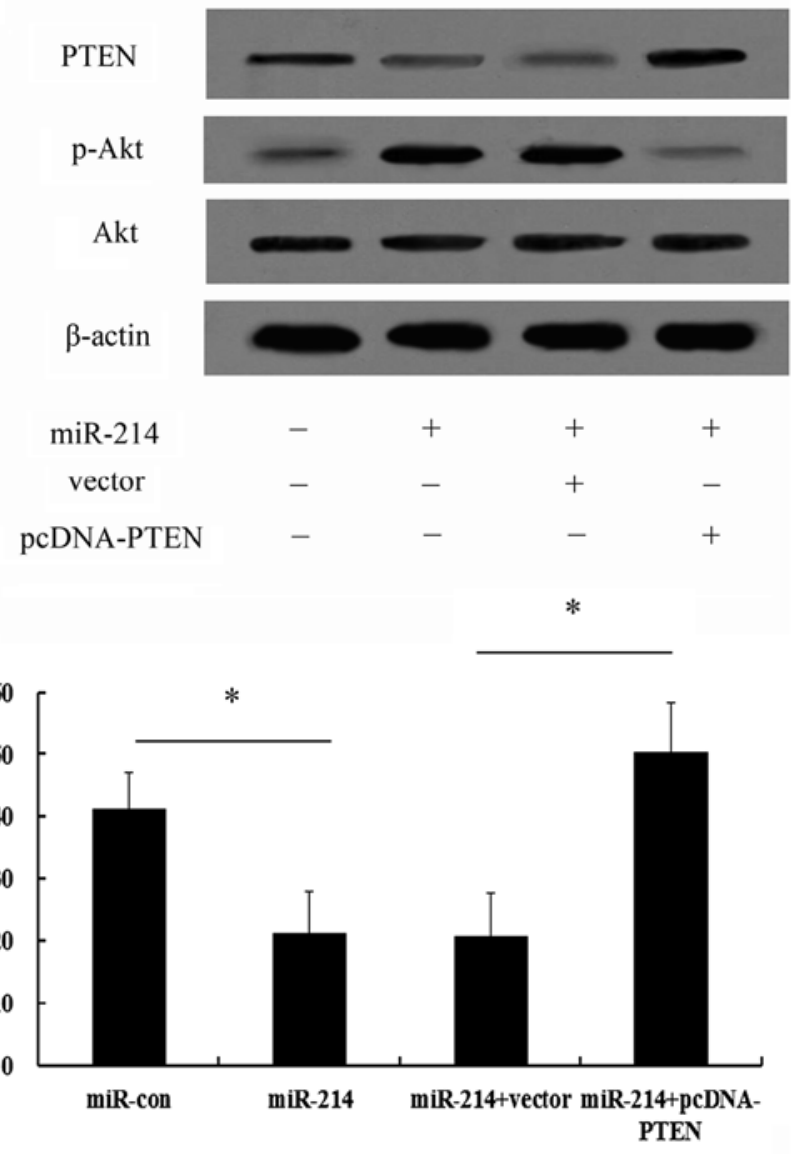

Figure 6. PTEN is responsible for the promoting effects of miR-214 on cell survival and resistance to apoptosis through PI3K/Akt signaling. (A and B) The effect of miR-214 on PTEN protein levels was determined by western blot analysis. (C) Alignment of miR-214 with PTEN at the 3' untranslated region (3'UTR) shown by bioinformatics analysis. The corresponding mutation in the 3'UTR of PTEN is also illustrated. (D) Following transfection with reporter vectors containing wild-type (wt) or mutant (mut) PTEN-3'UTR, the relative luciferase expression was normalized to Renilla activity. (E) The effect of PTEN on the miR-214-induced activation of PI3K/Akt was performed by transfection with pcDNA-PTEN lacking 3'-UTR. Simultaneously, the corresponding effects of miR-214 on (F) cell viability and $(\mathrm{G})$ resistance to apoptosis were also investigated. ${ }^{*} \mathrm{P}<0.05$.

sensitized the miR-214-expressing MCF-7 cells to apoptosis induced by serum starvation (Fig. 6G). Therefore, these data further validate that the PTEN/Akt pathway is a major target of miR-214 and that it is largely responsible for miR-214-mediated cell survival and anti-apoptotic effects.

\section{Discussion}

Breast cancer ranks as the leading cause of cancer-related mortality in women worldwide $(1,2)$. A substantial increase in the global incidence rates of breast cancer has been documented over the past few years. Therefore, elucidating the molecular mechanisms involved in the progression of breast cancer is pivotal for the development of therapies for breast cancer. This study presents the important finding that the expression of miR-214 was upregulated in several breast cancer cell lines compared with that in the non-malignant breast epithelial cell line, MCF-10A. Additionally, the overexpression of miR-214 markedly enhanced cell viability and promoted resistance to apoptosis. Furthermore, miR-214 was shown to be involved in the modulation of PI3K/Akt signaling by directly targeting PTEN. Taken together, these results suggest that miR-214 plays a critical role in the development and progression of breast cancer. 
miRNAs are endogenous, single-stranded, non-coding RNAs which are approximately 22 nucleotides in length and may suppress the post-transcriptional expression of target genes and are consequently involved in the regulation of various cellular processes, e.g., cell apoptosis, differentiation, development and metastasis $(9,18,19)$. Growing evidence has reinforced the fact that miRNAs are frequently deregulated and aberrantly expressed in certain types of cancer, by acting as either tumor suppressors $(9,20)$ or oncogenes $(21,22)$, and that they play a central role in carcinogenesis. Among these miRNAs, miR-214 has attracted increasing attention due to its critical role in the development of different types of cancer $(8,10)$; however, accumulating evidence has confirmed that miR-214 exerts various, even inverse, effects in different types of cancer. For example, miR-214 has been shown to function as an oncogene in melanoma to mediate tumorigenesis (8), whereas it acts as an anti-oncogene in hepatoma (10). Nevertheless, the roles of miR-214 in breast cancer remain unclear, as well as the underlying mechanisms. In this study, miR-214 expression was significantly elevated in four human breast cancer cell lines in contrast to its expression level in the non-malignant breast epithelial cell line, MCF-10A, which is consistent with the finding that miR-214 is expressed at higher levels in the serum of breast cancer patients compared with that in healthy patients or those with benign breast disease (13). Moreover, the overexpression of miR-214 markedly enhanced cell viability and abrogated cell apoptosis triggered by serum starvation, indicating a pivotal role of this miRNA in breast cancer cell growth. Consequently, these data suggest that miR-214 acts as a novel oncogene which regulates the progression of breast cancer.

It is widely accepted that carcinogenesis often results from the dirsupted balance between cell growth and programmed cell death (i.e., apoptosis). The PI3K/Akt signaling pathway has been proven to be involved in the above-mentioned processes and even other processes, and its expression is frequently disrupted in human cancer $(15,23)$. The abnormal activation of this pathway has been corroborated by epidemiological and experimental studies as a critical step toward the initiation and maintenance of human tumors. Previous research has demonstrated that PI3K/Akt triggers a cascade of multiple signals that regulate cancer cell proliferation, invasion, metastasis, survival as well as affecting prognosis $(24,25)$. Thus, in this study, to explore the mechanisms involved in the miR-214-mediated increase in cell viability and resistance to apoptosis, we examined the involvement of PI3K/Akt signaling in these processes. In accordance with our hypothesis, the overexpression of miR-214 evidently induced the activation of p-Akt. It is generally believed that cyclin D1, p27 and Bcl-2 are common downstream molecules of the PI3K/Akt pathway, which are all associated with cell proliferation and apoptosis $(26,27)$. The consistently higher expression of cyclin D1 and the anti-apoptotic protein, Bcl-2, was observed in the cells transfected with miR-214 mimics; however, the expression of the cell cycle inhibitory protein, p27, was downregulated. The above-mentioned results confirmed that the upregulation of miR-214 induced the activation of the PI3K/Akt pathway. Further mechanistic analysis validated that pre-treatment with the inhibitor, LY294002, notably blocked the activation of the PI3K/Akt pathway induced by miR-214. Simultaneously, the promoting effects of miR-214 on cell viability and its protective effects against apoptosis were markedly abrogated by pre-treatment with LY294002. Taken together, these results suggest that miR-214 positively promotes breast cancer cell growth by regulating PI3K/Akt signaling.

A number of studies have confirmed that the dysregulation of PTEN occurs in various types of cancer and that it plays a role as a tumor suppressor in the progression of cancer $(28,29)$. Recently, PTEN has been recognized as a negative regulator of PI3K/Akt signaling $(16,17)$. In ovarian cancer, the decrease in PTEN expression has been shown to induce the activation of the PI3K/Akt pathway (30). In the present study, the overexpression of miR-214 markedly decreased PTEN expression. Further bioinformatics analysis indicated that there was a conservative binding site of miR-214 in the 3'UTR of PTEN. Importantly, luciferase activity was markedly reduced when the cells were transfected with the PTEN-3'UTR-containing luciferase reporter system, but not in the PTEN-3'UTR-mutation groups, indicating that PTEN is a direct target of miR-214. Further mechanistic analysis validated that the miR-214-mediated activation of the PI3K/Akt pathway was markedly abrogated following transfection with pcDNA-PTEN lacking 3'UTR, implying that miR-214 induced the activation of the PI3K/Akt signaling pathway by directly targeting PTEN. Of note, the expression of PTEN significantly abrogated the protective effects of miR-214 on cell survival and resistance to apoptosis. Therefore, these data further confirm that miR-214 regulates cell survival and exerts anti-apoptotic effects mainly by targeting the PTEN-PI3K/Akt pathway.

In conclusion, the present study observed an upregulation of miR-214 in breast cancer cell lines. Importantly, miR-214 enhanced cell viability and promoted resistance to apoptosis by directly targeting PTEN-PI3K/Akt signaling, which ultimately facilitates the development of breast cancer. Consequently, pharmaceutical interventions targeting miR-214 may provide a promising therapeutic strategy for the treatment of breast cancer.

\section{References}

1. Jemal A, Bray F, Center MM, Ferlay J, Ward E and Forman D: Global cancer statistics. CA Cancer J Clin 61: 69-90, 2011.

2. Benson JR and Jatoi I: The global breast cancer burden. Future Oncol 8: 697-702, 2012

3. van Rooij E and Olson EN: MicroRNA therapeutics for cardiovascular disease: opportunities and obstacles. Nat Rev Drug Discov 11: 860-872, 2012.

4. Dai R and Ahmed SA: MicroRNA, a new paradigm for understanding immunoregulation, inflammation, and autoimmune diseases. Transl Res 157: 163-179, 2011.

5. Huang J, Lyu H, Wang J and Liu B: MicroRNA regulation and therapeutic targeting of survivin in cancer. Am J Cancer Res 5: 20-31, 2015.

6. Cheng H, Zhang L, Cogdell DE, Zheng H, Schetter AJ, Nykter M, Harris CC, Chen K, Hamilton SR and Zhang W: Circulating plasma miR-141 is a novel biomarker for metastatic colon cancer and predicts poor prognosis. PLoS One 6: e17745, 2011.

7. Fornari F, Milazzo M, Chieco P, Negrini M, Marasco E, Capranico G, Mantovani V, Marinello J, Sabbioni S, Callegari E, et al: In hepatocellular carcinoma miR-519d is up-regulated by 553 and DNA hypomethylation and targets CDKN1A/p21, PTEN, AKT3 and TIMP2. J Pathol 227: 275-285, 2012.

8. Penna E, Orso F, Cimino D, Tenaglia E, Lembo A, Quaglino E, Poliseno L, Haimovic A, Osella-Abate S, De Pittà C, et al: microRNA-214 contributes to melanoma tumour progression through suppression of TFAP2C. EMBO J 30: 1990-2007, 2011. 
9. Yamada Y, Hidaka H, Seki N, Yoshino H, Yamasaki T, Itesako T, Nakagawa $M$ and Enokida $\mathrm{H}$ : Tumor-suppressive microRNA-135a inhibits cancer cell proliferation by targeting the c-MYC oncogene in renal cell carcinoma. Cancer Sci 104: 304-312, 2013.

10. Shih TC, Tien YJ, Wen CJ, Yeh TS, Yu MC, Huang $\mathrm{CH}$, Lee YS, Yen TC and Hsieh SY: MicroRNA-214 downregulation contributes to tumor angiogenesis by inducing secretion of the hepatoma-derived growth factor in human hepatoma. J Hepatol 57: 584-591, 2012.

11. Wang Z, Cai H, Lin L, Tang M and Cai H: Upregulated expression of microRNA-214 is linked to tumor progression and adverse prognosis in pediatric osteosarcoma. Pediatr Blood Cancer 61: 206-210, 2014.

12. Wang J, Li J, Wang X, Zheng $\mathrm{C}$ and $\mathrm{Ma} \mathrm{W}$ : Downregulation of microRNA-214 and overexpression of FGFR-1 contribute to hepatocellular carcinoma metastasis. Biochem Biophys Res Commun 439: 47-53, 2013.

13. Schwarzenbach H, Milde-Langosch K, Steinbach B, Müller V and Pantel K: Diagnostic potential of PTEN-targeting miR-214 in the blood of breast cancer patients. Breast Cancer Res Treat 134: 933-941, 2012.

14. Li B, Han Q, Zhu Y, Yu Y, Wang J and Jiang X: Down-regulation of miR-214 contributes to intrahepatic cholangiocarcinoma metastasis by targeting Twist. FEBS J 279: 2393-2398, 2012.

15. Osaki M, Oshimura M and Ito H: PI3K-Akt pathway: its functions and alterations in human cancer. Apoptosis 9: 667-676, 2004

16. Moon S-H, Kim D-K, Cha Y, Jeon I, Song J and Park K-S PI3K/Akt and Stat3 signaling regulated by PTEN control of the cancer stem cell population, proliferation and senescence in a glioblastoma cell line. Int J Oncol 42: 921-928, 2013.

17. Abdulaziz S, Al-Shahid M and Al-Thenayan E: A 49-year-old man with acute pulmonary hypertension post lung transplantation. Chest 144: 704-707, 2013.

18. Baumjohann D, Kageyama R, Clingan JM, Morar MM, Patel S, de Kouchkovsky D, Bannard O, Bluestone JA, Matloubian M, Ansel KM and Jeker LT: The microRNA cluster MIR-17 92 promotes TFH cell differentiation and represses subset-inappropriate gene expression. Nat Immunol 14:840-848, 2013.

19. Zhou Y, Xiong M, Niu J, Sun Q, Su W, Zen K, Dai C and Yang J: Secreted fibroblast-derived miR-34a induces tubular cell apoptosis in fibrotic kidney. J Cell Sci 127: 4494-4506, 2014.

20. Zha R, Guo W, Zhang Z, Qiu Z, Wang Q, Ding J, Huang S, Chen T, Gu J, Yao M and He X: Genome-wide screening identified that miR-134 acts as a metastasis suppressor by targeting integrin $\beta 1$ in hepatocellular carcinoma. PLoS One 9: e87665, 2014.
21. Yau WL, Lam CSC, Ng L, Chow AK, Chan ST, Chan JY, Wo JY, Ng KT, Man K, Poon RT and Pang RW: Over-expression of miR-106b promotes cell migration and metastasis in hepatocellular carcinoma by activating epithelial-mesenchymal transition process. PLoS One 8: e57882, 2013.

22. Josson S, Gururajan M, Hu P, Shao C, Chu GY, Zhau HE, Liu C, Lao K, Lu CL, Lu YT, et al: miR-409-3p/-5p promotes tumorigenesis, epithelial-to-mesenchymal transition, and bone metastasis of human prostate cancer. Clin Cancer Res 20: 4636-4646, 2014.

23. Slomovitz BM and Coleman RL: The PI3K/AKT/mTOR pathway as a therapeutic target in endometrial cancer. Clin Cancer Res 18: 5856-5864, 2012.

24. Yulyana Y, Ho IA, Sia KC, Newman JP, Toh XY, Endaya BB, Chan JK, Gnecchi M, Huynh H, Chung AY, et al: Paracrine factors of human fetal MSCs inhibit liver cancer growth through reduced activation of IGF-1R/PI3K/Akt signaling. Mol Ther 23: 746-756, 2015.

25. Vo BT, Morton D Jr, Komaragiri S, Millena AC, Leath C and Khan SA: TGF- $\beta$ effects on prostate cancer cell migration and invasion are mediated by PGE2 through activation of PI3K/AKT/mTOR pathway. Endocrinology 154: 1768-1779, 2013.

26. Yakes FM, Chinratanalab W, Ritter CA, King W, Seelig S and Arteaga CL: Herceptin-induced inhibition of phosphatidylinositol-3 kinase and Akt is required for antibody-mediated effects on p27, cyclin D1, and antitumor action. Cancer Res 62: 4132-4141, 2002.

27. Kumar P, Miller AI and Polverini PJ: p38 MAPK mediates $\gamma$-irradiation-induced endothelial cell apoptosis, and vascular endothelial growth factor protects endothelial cells through the phosphoinositide 3-kinase-Akt-Bcl-2 pathway. J Biol Chem 279: 43352-43360, 2004.

28. Song MS, Salmena L and Pandolfi PP: The functions and regulation of the PTEN tumour suppressor. Nat Rev Mol Cell Biol 13: 283-296, 2012.

29. Mulholland DJ, Tran LM, Li Y, Cai H, Morim A, Wang S, Plaisier S, Garraway IP, Huang J, Graeber TG and Wu H: Cell autonomous role of PTEN in regulating castration-resistant prostate cancer growth. Cancer Cell 19: 792-804, 2011.

30. Lee S, Choi EJ, Jin C and Kim DH: Activation of PI3K/Akt pathway by PTEN reduction and PIK3CA mRNA amplification contributes to cisplatin resistance in an ovarian cancer cell line. Gynecol Oncol 97: 26-34, 2005. 